\section{Thickness constraints on the icy shells of the galilean satellites from a comparison of crater shapes}

\section{Paul M. Schenk}

Lunar and Planetary Institute, 3600 Bay Area Boulevard, Houston, Texas 77058, USA

A thin outer ice shell on Jupiter's large moon Europa would imply easy exchange between the surface and any organic or biotic material in its putative subsurface ocean ${ }^{1-4}$. The thickness of the outer ice shell is poorly constrained, however, with modeldependent estimates ranging from a few kilometres ${ }^{5,6}$ to ten or more kilometres? ${ }^{7}$. Here I present measurements of depths of impact craters on Europa, Ganymede and Callisto that reveal two anomalous transitions in crater shape with diameter. The first transition is probably related to temperature-dependent ductility of the crust at shallow depths $(7-8 \mathrm{~km}$ on Europa). The second transition is attributed to the influence of subsurface oceans on all three satellites ${ }^{3,8,9}$, which constrains Europa's icy shell to be at least $19 \mathrm{~km}$ thick. The icy lithospheres of Ganymede and Callisto are equally ice-rich, but Europa's icy shell has a thermal structure about 0.25-0.5 times the thicknesses of Ganymede's or Callisto's shells, depending on epoch. The appearances of the craters on Europa are inconsistent with thin-ice-shell models ${ }^{1}$ and indicate that exchange of oceanic and surface material could be difficult.

The thickness of Europa's ice shell controls whether exchange between Europa's surface and its putative water ocean (including exposure of possible organic materials to sunlight' ${ }^{\prime}$ is possible, and in what manner. A thick shell (>10 km thick) would be susceptible to solid-state convection and overturn ${ }^{10}$, whereas a thin ice shell (only a few kilometres thick) would be vulnerable to crack-through and melt-through from below ${ }^{1.5}$. Efforts to constrain the thickness of Europa's icy shell through geologic ${ }^{5-7}$ and geophysical ${ }^{3,4}$ investigations have thus far failed to produce a consensus because of controversy over the origin of geologic structures ${ }^{1,2}$ and weak model constraints. Impact craters (Fig. 1) sample planetary lithospheres over a range of depths and offer direct probes into Europa's icy shell. Crater morphology is influenced by planetary surface gravity ${ }^{\prime \prime}$ and lithospheric properties ${ }^{12,13}$. Surface gravity on the three icy galilean satellites and the Moon are similar (Europa, $132 \mathrm{~cm} \mathrm{~s}^{-2}$; Ganymede, $143 \mathrm{~cm} \mathrm{~s}^{-2}$; Callisto, $124 \mathrm{~cm} \mathrm{~s}^{-2}$; the Moon, $162 \mathrm{~cm} \mathrm{~s}^{-2}$ ), so differences in crater morphology can be attributed to compositional or rheological differences.

Moore et $a l .^{14,15}$, in their initial survey of Galileo observations of crater morphologies on Europa, found that the largest craters (Fig. 1) are morphologically distinct from and shallower than similarsized craters on Ganymede and Callisto (Fig. 1). They concluded that these craters may have excavated into either a global subsurface ocean or a ductile zone in the lower shell, but did not conduct a systematic analysis of crater dimensions. Recent attempts to model impact melt production suggest that the ice shell may be at least 3 $4 \mathrm{~km}$ thick ${ }^{\mathrm{kn}}$, but this does not constitute a definitive test of the "thin crust, thick crust' debate.

Depth/diameter $(d / D)$ data for fresh, undegraded, craters on the Moon and other planets follow well-defined trends and feature two breaks in slope $e^{11,17}$. These breaks, or transitions, correlate with morphologic transitions from simple bowl-shaped to complex morphologies (featuring slump terraces and uplifted central peaks) and from complex craters to ringed basins (see, for example, ref. 18). For comparison, I have catalogued all craters on Ganymede and Callisto that are over $30 \mathrm{~km}$ across, and over $1 \mathrm{~km}$ across on Europa. I have also measured the depths of fresh (unmodified and uncelaxed) craters from $100 \mathrm{~m}$ to $170 \mathrm{~km}$ in diameter on the icy galilean satellites (Fig. 2) using Voyager and Galileo images. Three transitions in crater shape have been identified on each satellite, but two of these are anomalous and occur at different diameters on Europa than on Ganymede and Callisto.

Transition I (from simple to complex morphology) is similar on all three satellites (Fig. 2) and can be associated with regular crater modification processes ${ }^{18}$. Transition II on these satellites correlates with anomalous changes in complex crater dimensions and morphology. On Ganymede and Callisto, this occurs at $D \approx 26 \mathrm{~km}$, where crater depths begin to decrease slightly $(d \approx 1.1 \pm 0.3 \mathrm{~km})$ and are shallower than predicted from extrapolation of the $d / D$ curve for complex craters (Fig. 2a, b). This change coincides with the morphologic transition from central peak craters to central pit and dome craters ${ }^{19}$ (Fig. 1). Transition II on Europa occurs at $D:=8 \mathrm{~km}$, where complex craters on Europa diverge from their galilean siblings and become increasingly shallower than similarsized complex craters on Ganymede and Callisto (Fig. 2c). Central peaks still occur but crater rim and peak morphology becomes progressively disrupted with diameter (Fig. 1).

Transition III is associated with a sharp reduction in crater depths and the development of anomalous impact morphologies. On Ganymede and Callisto, many large central dome craters have poorly developed or non-existent rims (Fig. 1). Floors of these 'anomalous dome craters' (Fig. 1) are similar to or elevated a few $100 \mathrm{~m}$ above surrounding plains. Rim-to-floor depths are negligible to about $500 \mathrm{~m}$ (Fig. 2b). Anomalous dome morphologies in older craters (based on superposed crater densities) occur at $D>60 \mathrm{~km}$, indicating a Transition III diameter as low as $60 \mathrm{~km}$ in post-brightterrain formation times. For younger central dome craters only those larger than about $150 \mathrm{~km}$ in diameter have anomalous murphologies, suggesting that Transition III diameters on Ganymode and Callisto have increased to about $150 \mathrm{~km}$ at present.

Transition III on Europa occurs at $D \approx 30 \mathrm{~km}$ (Fig. 2c) and correlates with an abrupt change from modified central peak to multiring morphologies between 27 and $33 \mathrm{~km}$ crater diameter. The twi largest impact structures, Callanish and Tyre $(D \approx 33$ and $41 \mathrm{~km}$, respectively, as determined by scaling from mappable ejecta deposits $\left.\mathrm{s}^{20}\right)$ are characterized by several $(>5)$ concentric ridges and

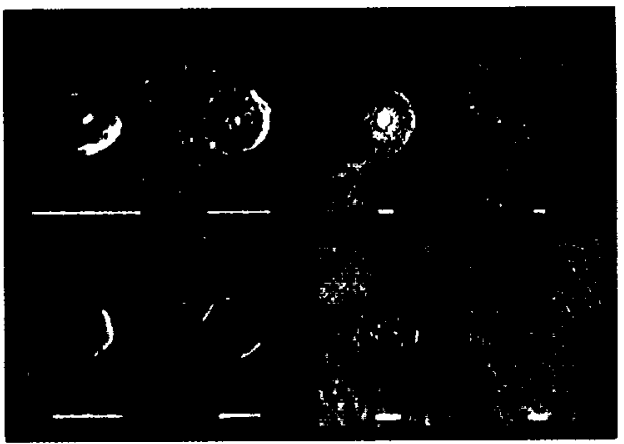

Figure 1 Impact crater landforms on the icy galilean satellites, representing the major mo phologic types. In order of increasing diameter from left to right crater types are, for Gan iymede and Callisto (top row): central peak craters $(D=18 \mathrm{~km})$, central pit craters $(D=30 \mathrm{~km})$, central dome craters (Enkidu, $D=121 \mathrm{~km}$ ) and anomalous dome craters $(D=138 \mathrm{~km}$ ). For Europa (bottom row): central peak craters $(D=8 \mathrm{~km})$, modified certrat pit craters (Grainne, $D=14 \mathrm{~km}$ ), anomalous central peak craters (Pwyll, $D:=27 \mathrm{~km}$ ), and multiring basins (Tyre, $D=41 \mathrm{~km}$ ). Images have been scaled so that craters appear to have the same diameter. Scale bars are $30 \mathrm{~km}$ for Ganymede and Ca:iisto (top row), and $10 \mathrm{~km}$ for Europa (bottom row). Images for Pwyll and Tyre and the unilamed Ganymede basin have been colour-coded to represent topography (red, high; blue, low colour scale bars represent relative elevation: 0 to $500 \mathrm{~m}$ for Europa craters and 0 1) $2,000 \mathrm{~m}$ for Ganymede). Note the progressively more complex central peak morphology with increasing crater diameter. The nominal rim location for Tyre lies within the nnermost of the prominent topographic ridges. The Sun is from the left in all images. 
graben $^{15}$, but no central structures, depressions or rim scarps can be identified within the nominal crater (Fig. 1). Maximum effective crater depths, inferred from isolated ridges near the rim location and subtle topography across the floor, are between 50 and $100 \mathrm{~m}$ (Figs 1,20 ). These structures differ sharply from terrestrial planet multiring basins which have only 3-5 ring scarps and topography greater than $4 \mathrm{~km}$ (for example, refs 13,17 ).

The rollover and precipitous drop in crater depths with diameter for the galilean satellites (Fig. 2) is unprecedented among the planets. That this is not due to long-term post-impact flattening
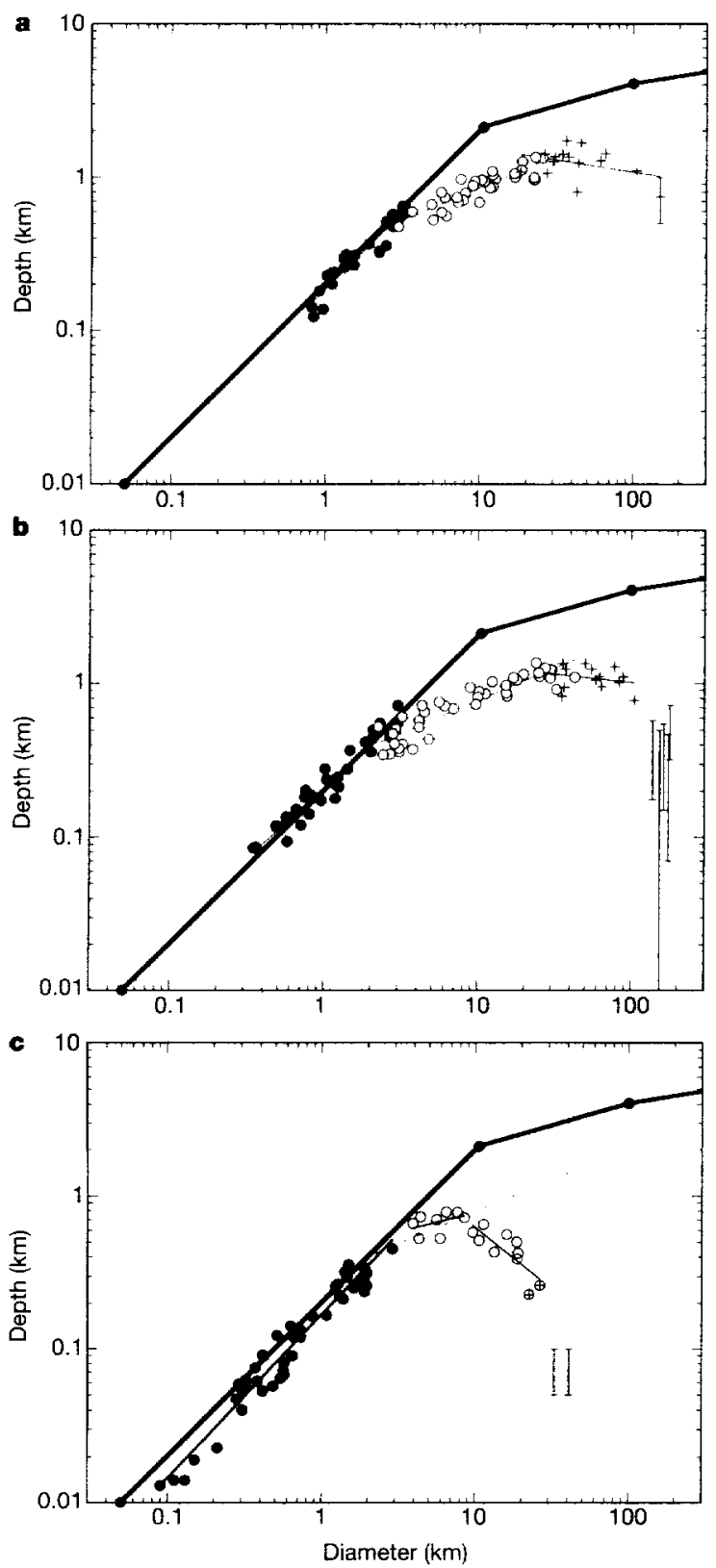

Figure 2 Depth/diameter measurements for fresh impact craters on the icy galilean satellites. Data are plotted separately for Callisto (a), Ganymede (b) and Europa (c). The heaw dark line is for lunar craters ${ }^{11.17}$. The thin lines are least-squares fits through data. Craters plotted are simple craters (solid dats), complex (central peak) craters (open circies). central pit and central dome craters (crosses), and anomalous dome and multiring basins (error bars). For Europa, the modified central peak craters Mannann'an and Pwyll are plotted as circles with crosses. The dashed line in $\mathbf{c}$ is the least-squares fit through complex craters on Ganymede for comparison with complex craters on Europa. Note the sparser data set for Callisto. The error bar for the largest central dome crater on Callisto is typical for all plotted data by creep (that is, viscous relaxation) is suggested by the youth of the craters studied (some have well-preserved bright rays) and by their misshapen or absent central structures and rims. Relaxation and volcanism may flatten or bury topography but are unlikely structurally to disrupt rims and central peaks (see ref. 21). Rugged relief on crater floors (Fig. 1) is also inconsistent with the idea that craters formed within a giant pool of impact melt ${ }^{10}$. Thus, differences in internal structure between the icy satellites and the Moon, and between the icy galilean satellites, are the most plausible explanation for the unusual shapes of larger craters on these satellites. Warm ice probably at shallow depths within these satellites is notoriously weaker than common silicates and the very cold brittle ice near their surfaces (see ref. 22). I propose that Transitions II and III reveal at least two such temperature-induced rheologic transitions or phase changes with depth on all three icy galilean satellites, but that these transitions are much shallower on Europa. Inherently weaker ice at greater depths would be progressively less able to support the topography associated with larger transient craters several kilometres deep ${ }^{23}$, leading to enhanced structural collapse.

Impact into rheologically layered targets is imprecisely understood. Studies of terrestrial and lunar craters ${ }^{18,24}$ and recent modelling ${ }^{2.3}$ of crater floor rebound suggest that modification from simple to complex morphology (by floor rebound and rim collapse) involves a quasi-hemispherical zone around the transient (premodification) crater ${ }^{1 *}$ and that the modification process and final crater shape begins to be unduly influenced when an anomalously weak layer is roughly 1 to 1.3 times as deep as the transient crater width. The McKinnon and Schenk ${ }^{25}$ scaling relationship for icy satellite craters is used to estimate transient crater dimensions (to within approximately $10 \%$ ) from the observed crater diameters. On Ganymede and Callisto, Transition II craters $(D \approx 26 \mathrm{~km})$ scale to transient craters about $16.5 \mathrm{~km}$ wide for a depth to the first anomalous layer of $\sim 16-22 \mathrm{~km}$. On Europa, Transition II craters $(I) \approx 8 \mathrm{~km}$ ) scale to transient craters about $6 \mathrm{~km}$ wide, for a layer depth of $7-8 \mathrm{~km}$. On Ganymede and Callisto, Transition III craters $(D \approx 150 \mathrm{~km}$ ) scale to transient craters about $80 \mathrm{~km}$ wide, for a depth to the second anomalous layer of $80-105 \mathrm{~km}$ (the older Transition III diameter of $\sim 60 \mathrm{~km}$ translates to transient diameters of around $35 \mathrm{~km}$, and a layer depth of $35-45 \mathrm{~km}$ ). On Europa, Transition III craters $(D \approx 30 \mathrm{~km})$ scale to transient craters about $19 \mathrm{~km}$ wide, and a depth to the second transition of $19-25 \mathrm{~km}$.

Transition II is interpreted to represent a temperature-dependent rheologic change with depth. The preservation of prominent (albeit modified) central peaks in complex craters not yet at Transition II is consistent with solid-state material at the base of the transient crater $^{14,16}$. Rounded central domes, modified central peaks and degraded rim topography in craters larger than those at Transition II (Fig. 1) are all consistent with coherent but very warm ductile ice in the lower ice shell (see ref. 19).

Galileo tracking data indicate that the interior of Ganymede is probably strongly differentiated with an ice-rich lithosphere and mantle, whereas Callisto is not fully differentiated and may or may not have an ice-rich outer lithosphere ${ }^{26,27}$. The indistinguishable d/D statistics for craters on Ganymede and Callisto (Fig. 2) indicate that internal composition and thermal structure of these two satellites are similar, and that Callisto also has an ice-rich outer shell at least several tens of kilometres thick.

Galileo magnetometer observations are consistent with internal oceans currently within both Ganymede and Callisto at depths of $150-200 \mathrm{~km}$ but possibly shallower ${ }^{x, 4}$. If Transition III is a detection of oceans on Ganymede and Callisto, they must be at least $80 \mathrm{~km}$ deep at present. Changes in morphology and transitions on Ganymede and Callisto as described here may record changes in lithospheric thermal properties ${ }^{28}$ and possibly ocean depths over time.

Transition III to multiring morphologies on Europa may indicate the beginning of the influence of a global ocean ${ }^{15}$. The unusual multiring morphologies on icy satellites are probably related to 
impact into a thin brittle lithosphere overlying a fluid or a ductile layer that behaves as a fluid during crater collapse". The apparent abruptness of Transition III on Europa (Figs 1,2) is consistent with a sharp phase change at depth, such as an ice-liquid interface, but may also represent a rapid transition to the hot base of the ice shell where the ice is near melting and very ductile. It seems that the base of the icy shell is not much deeper, but the inferred Transition III depth at least indicates a minimum ice shell thickness of around 19$25 \mathrm{~km}$. Europa's known impact craters are unlikely to excavate ocean material directly to the surface, however (unless the material is trapped in isolated shallow reservoirs). The largest crater, Tyre, excavates down to only about $3 \mathrm{~km}(\sim 30-40 \%$ of the transient crater depth $\left.{ }^{18}\right)$, or around $15 \%$ of the minimum shell thickness.

Systematic mapping of impact crater shape and morphology provides a robust (and free) means of sampling icy satellite interiors. Ice rheology is dependent on temperature ${ }^{22}$ and these transition diameters provide new constraints on the comparative thermal structure of these satellites. Transitions II and III are 2-4 times shallower on Europa than on Ganymede or Callisto, indicating that the rheologic structure of Europa's outer shell is similarly thinner than on Ganymede and Callisto. As heat flow scales roughly linearly with the thickness of the stagnant non-convecting lid ${ }^{10}$ (possibly represented by Transition II), heat flow should to first order also be 2-4 times higher on Europa, depending on epoch. Transition III constrains Europa's ice shell to be at least $19-25 \mathrm{~km}$ thick, consistent with that required for convection to proceed within the ice shell ${ }^{10}$ and favouring diapirism of the lower shell for the origin of ovoid features and chaos terrains (refs 7, 29 and P.S. and R. Pappalardo, manuscript in preparation). The minimum shell thickness strongly supports thick ice shell models in general, and associated interpretations of geologic features ${ }^{27.29}$; it may also indicate the depth to Europa's ocean, or at least the beginning of the hot basal layer of the floating ice shell.

\section{Methods}

Crater depths have been measured from Cialileo and Voyager data using three lechniques: stereo digital clevation models (DEMs), photoclinometry DEMs, and shadow length measurements. Stereo DEMs resolve only the large craters Pwyll, Mannann'an, and Cilix (diameters $19-27 \mathrm{~km}$ ) on Furopa, and several anomalous dome craters on (ianymede. photodinometry in two horizontal dimensions can be used with single low-sun images to map topography from relative brightness. My photoclinometry technique includes the use of low-phase-angle images to model local albedo, thus reducing or eliminating one of the major systematic sources of error in photoctinometry. This technique is used here primarily to confirm the stereo and shadow measurements, but is the primary source of topographic data for the multiring basins Callanish and Tyre. Photoclinometry was also used to map topography across additional anomalous dome craters on Canymede. supporting the conclusion that they have raised floors based on limited stereo data described atove. I also use low-resolution controlled stereo DEMs to control the longwavelength component of high-resolution photoclinometry DFMs. This was especially useful for P'wyll (Fig. 1). Height measurements based on triangulation of shadow lengths are used for all craters on Furopa, Ganymede and Callisto smaller than $\sim 15 \mathrm{~km}$ across. Depth measurement errors include systematic errors due to technique and those due to variations in topography along the rim crest. Systematic errors rarely exceed $10 \%$.

Variations in rim height approach about $100 \mathrm{~m}$ for large craters such as Pwyll. These data supercede the depth/diameter statistics for Ganymede and Callisto based on lower-

resolution Voyager data ${ }^{2,49}$; no Voyager-based measurements were possible for craters on Europa.

Received 21 January; accepted 8 April 2002.

I. Cireenberg. R. Tides and the biosphere of liuropi. Am. Sit 90, 48-55(2002)

2. Pappalardo. R. a al. Does Furopa have a subsurface ocean? Esaluation of the geologic evidence I. (ievpliys. Res. 104, 24015-24056!1999!

3. Kivelson, M. et al (iatileo magnetometer measurements: A si runger case for a subsurface ocean on:

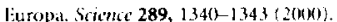

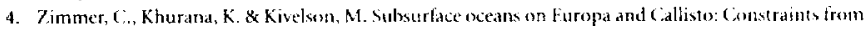
Galilco magnetonteter observations. Lam $147,329,3,47,2000\rfloor$

5. Greenberg. R. Gieissler, P., Juft, B. R. \& Hoppa, C. Habitability of Europa's crust: The role of tidalIectonic processes. J. (iemphys. Ros. 105, 1755:-17562 (2000).

6. Hoppa, C., Tifts, B. R., Gicentorg, R. \& Gicissler, P. Formation of cycloidal leatures un E.uropa. Science 2B5, $1899-1903(1949)$.

7. Rathbun, I., Musser, (i. \& Squyres, S. Ice diapirs on Europa: Implications for liquid water. (icophys. Res. l.t1. $25,4157-4160)(1998)$.

8. Kivelson, M. Khurana, K. \& Volwerk, M. Janus (in the press).

9. Kivelson, M. of al. Europa and (Clllsto: Induced or intrinsit liekds in a periodically varying plasma envirumment. /. Giophys. Res 104, 410994625 (1949)1.
1.. Mikinnon, W. Convective instabifity in Furopa's floating ice shell Geophys. Res. Lett. 26, 9\$1-954 (1999)

Pike, R. in Mercury teds Vilas, F., Chapman, C, \& Malthews, M.1 36.5 .273 i L iniv. Arizona Press Tucsom, l 1 YkR

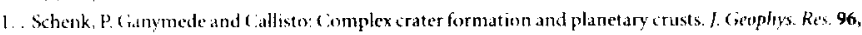
$15635-1566+11991)$.

1. Mckinuon, W. \& Metosh, H. I. Fvolution of planetary lithospheres: Evidence from multiring hasins on (janymede and (allisto. Lion $44,454-471$ (1998).

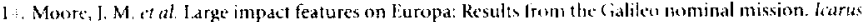
$135,127-14,(1998)$

. Moore, J. M. ct al. Large impact features on Furopa: Results from the Galileu ( FM m mssimn. Lams 151, $93-111(20) 1)$.

11. Turtlc, E. \& Picrazzo, E. Thickness of a Europan ice shell from impat craler simulations. Sotemer 294 $1.326-1.328(2001)$.

1. Willians, K. \& Zuher, M. Measuremenl and analysis of lunar basin depth from Clementine data. larm 131, 107-122(1998)

1:. Melush, II. I. Impat Cratering (Oxford Press, (oxford. 1989)

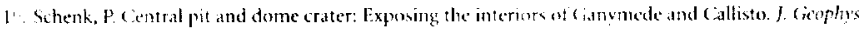
Res. 98, $7475.7498\{1993\}$.

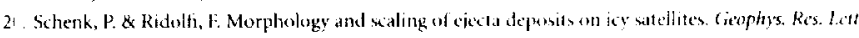
(in the press).

2. Dembard, A. \& Bckiunon, W. Long-lerm retention of impact iratcr tepongraphy on Ganymede. (jetoplys. Res. Lett. 27, 3663-36th $\{2000\}$.

2. Durham, W. Kirby $\$$ \& Stern, I. Creep of water ises under planctary conditions: A compilation. 1. (rotolys. Re's. 102, $10293-10,301(1997)$

2 Turtle, 1. \& Lanus, B. Numerical simulations of craler excavation ind collatye on Europa

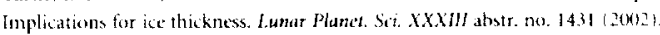

2. Melesh, H. I. \& (iaffine, E. Acoustic fluidization and the xale dependence of impat crater morphology. I. Cieophys. Res. 88, A830-A834 (1983\}.

2. McKinnon. W. \& Schenk, P. Estimates of comet fragtment masses from inpate t rater chains on Calliste

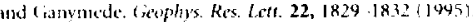

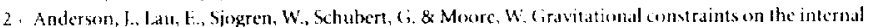
sirucure of (janymede. Nature 384, 541-543, (1946).

2 Anderson, J. at al. Shape, mean radius, gravity lield and internal structure of ( allistr). Learus 153 $157-161 ! 2001)$.

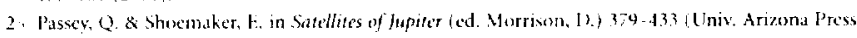
luesm, 1982).

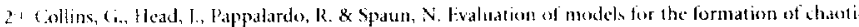
terrdin on Europa. f. Cieophys. Res. 105, 1709-1716 (2000).

\section{Acknowledgements}

This work was supported by NASA Planetary Geology and Cicophysics.

\section{Competing interests statement}

7 tec author deitares that he has no competing financial interests.

Correspondence and requests for materials should be addressed to the author (2) mail: schenk(ux)!pi usra.edu) 
